\title{
Terminal-Modality-Based Joint Call Admission Control Algorithm for Fair Radio Resource Allocation in Heterogeneous Cellular Networks
}

\author{
Olabisi E. Falowo \\ Department of Electrical Engineering, University of Cape Town, Cape Town, South Africa \\ Email: bisi@ieee.org
}

Received December 13, 2011; revised April 26, 2012; accepted May 11, 2012

\begin{abstract}
There is a problem of unfairness in allocation of radio resources among heterogeneous mobile terminals in heterogeneous wireless networks. Low-capability mobile terminals (such as single-mode terminals) suffer high call blocking probability whereas high-capability mobile terminals (such as quad-mode terminals) experience very low call blocking probability, in the same heterogeneous wireless network. This paper proposes a Terminal-Modality-Based Joint Call Admission Control (TJCAC) algorithm to reduce this problem of unfairness. The proposed TJCAC algorithm makes call admission decisions based on mobile terminal modality (capability), network load, and radio access technology (RAT) terminal support index. The objectives of the proposed TJCAC algorithm are to reduce call blocking/dropping probability, and ensure fairness in allocation of radio resources among heterogeneous mobile terminals in heterogeneous networks. An analytical model is developed to evaluate the performance of the proposed TJCAC scheme in terms of call blocking/dropping probability in a heterogeneous wireless network. The performance of the proposed TJCAC algorithm is compared with that of other JCAC algorithms. Results show that the proposed algorithm reduces call blocking/dropping probability in the networks, and ensure fairness in allocation of radio resources among heterogeneous terminals.
\end{abstract}

Keywords: Call Admission Control; Terminal Modality; Heterogeneous Terminals; Radio Access Technology; Call Dropping; Call Blocking; Markov Chain; Mobile Users

\section{Introduction}

The success of next generation networks will be derived from a combination of terminal heterogeneity and network heterogeneity [1]. Terminal heterogeneity refers to different types of terminals in terms of number of networks supported (modality), display size, energy consumption, portability/weight, complexity, etc. [1].

Network heterogeneity on the other hand refers to a combination of multiple wireless networks based on different access technologies (e.g. UMTS, EV-DO, LTE, WiMAX, etc.) coexisting in the same geographical area. This coexistence of different radio access technologies (RATs) in the same area necessitates joint radio resource management (JRRM) for enhanced QoS provisioning and efficient radio resource utilization. With joint radio resource management in NGWN, mobile users will be able to communicate through any of the available radio access technologies (RATs) and roam from one RAT to another, using multi-mode terminals (MTs) [2-8].

The joint call admission control (JCAC) algorithm is one of the RRM algorithms. The basic function of JCAC algorithms is to decide whether an incoming call can be accepted or not. They also decide which of the available radio access networks is most suitable to accommodate the incoming call.

Next generation networks are expected to support diverse types of terminals, such as mobile phones, personal digital assistants, iPads, netbooks, laptops, etc. Equipment manufacturers have come up with different terminals that possess a wide range of capabilities in order to take full advantage of heterogeneous wireless networks.

These mobile terminals will be able to access network services by choosing one of several RATs that are available in heterogeneous wireless networks. In order to achieve this purpose, terminals will have multiple access interfaces (multi-modality) or a dynamically reconfigurable access interface [1,9].

However, network operators cannot force all subscribers on their networks to suddenly change their singlemode mobile terminals to multi-mode terminals so as to enjoy the full benefits of heterogeneous wireless networks. Transitioning from using single-mode terminals to using J-mode ( $\mathrm{J}$ is the total number of available RATs) 
terminals by subscribers will be a gradual process. Consequently, mobile terminals with different capabilities such as single-mode, dual-mode, triple-mode, quad-mode, etc., will co-exist in next generation wireless networks. A combination of these terminals is referred to as heterogeneous mobile terminals in this paper.

For example, it was projected that 2G/2.5G/3G triplemode terminals would be available for most users in 2009-2010 [10]. With the current development in 3GPPLTE and WiMAX (IEEE 802.16e), quad-mode terminals will likely be available in the very near future. Meanwhile some subscribers in the heterogeneous wireless network will still retain their single-mode terminals. Figure 1 illustrates a heterogeneous wireless network supporting heterogeneous mobile terminals.

As shown in Figure 1, the heterogeneous wireless network consists of three RATs namely RAT 1, RAT 2, and RAT 3. Six mobile terminals $M t_{1}, M t_{2}, M t_{3}, M t_{4}, M t_{5}$, and $M t_{6}$ are shown in Figure 1. $M t_{1}$ and $M t_{2}$ are triplemode terminals that are supported by the three RATs. $M t_{3}$ and $M t_{4}$ are dual-mode terminals that are supported by RAT 1 and RAT 2. $M t_{5}$ and $M t_{6}$ are single-mode terminals that are supported by RAT 1 only.

Existing JCAC algorithms do not consider mobile terminal heterogeneity and specifically, mobile terminal modality, in making call admission decisions. In a heterogeneous wireless network supporting heterogeneous mobile terminals, if terminal modality is not considered in making call admission decisions, there will be a problem of unfairness in allocation of radio resources among the heterogeneous terminals in the network. Low-capability mobile terminals (such as $M t_{5}$ and $M t_{6}$ in Figure 1) will experience high call blocking probability whereas high-capability mobile terminals (such $M t_{1}$ and $M t_{2}$ in Figure 1) will experience very low call blocking probability, in the same heterogeneous wireless network. Thus, the low-capability terminals will be treated unfairly in the heterogeneous wireless network.

In order to reduce this problem of unfairness in allocation of radio resources among heterogeneous terminals in heterogeneous wireless networks, a terminal-modalitybased JCAC (TJCAC) scheme is proposed for heterogeneous wireless networks. The proposed TJCAC scheme is designed to simultaneously achieve the following objectives in heterogeneous cellular networks:

1) Ensure fairness in allocation of radio resources among heterogeneous mobile terminals;

2) Guarantee the QoS requirement of all admitted calls;

3) Prioritize handoff calls over new calls.

To the best my knowledge, this is the first work considering terminal modality in making joint call admission control decisions in heterogeneous wireless networks.

The contributions of this paper are twofold. Firstly, a

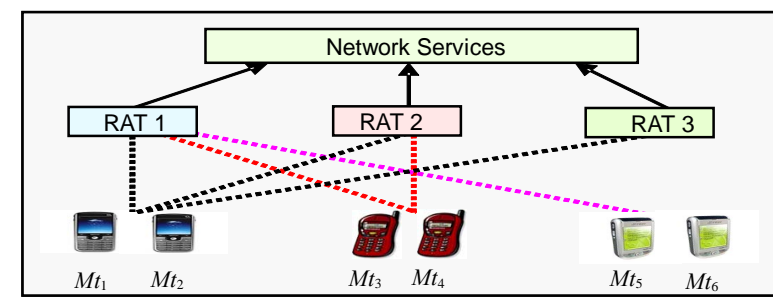

Figure 1. A three-RAT heterogeneous wireless network supporting heterogeneous mobile terminals.

terminal-modality-based JCAC scheme is proposed for heterogeneous wireless networks. Secondly, an analytical model is developed to evaluate the performance of the proposed TJCAC scheme in a heterogeneous cellular network.

The rest of this paper is organized as follows. In Section 2, existing RAT selection approaches in heterogeneous wireless networks are reviewed. The proposed terminal modality-based JCAC scheme is described in Section 3. Section 4 presents the heterogeneous network model. In Section 5, the heterogeneous terminal model is presented. An analytical model is developed for the proposed JCAC scheme in Section 6. In Section 7, the performance of the proposed JCAC scheme is evaluated through numerical simulations and results are presented.

\section{Existing RAT-Selection Approaches}

Some RAT selection approaches have been proposed in the literature [2,11-19]. These approaches namely, equal probability-RAT selection approach, service-class-based approach, load-based approach, service-cost-based approach, path-loss-based approach, and layer-based approach, are briefly reviewed in this section.

\subsection{Equal-Probability (or Random-Selection)- Based JCAC}

In this approach [2], when a new or vertical handoff call arrives, one of the available RATs is randomly selected for the call. The probability of selecting a particular RAT is $1 / \mathrm{J}$ where $\mathrm{J}$ is the total number of RATs in the heterogeneous wireless network. The advantage of this algorithm is that it is easy to implement. However, it has a high call blocking/dropping probability, and has the problem of unfairness in distribution of radio resources among heterogeneous mobile terminals.

\subsection{Service-Class-Based JCAC}

Service-class based JCAC algorithms [2,11] admit calls into a particular RAT based on the class of service, such as voice, video streaming, real-time video, web browsing, etc. This approach is based on the fact that different RATs are optimized to support different classes of service. The algorithm admits an incoming call into a RAT 
that can best support the service class of the call. Service-class-based JCAC algorithms may lead to highly unbalanced network load. Moreover, it has problem of unfairness in distribution of radio resources among heterogeneous mobile terminals.

\subsection{Load-Based JCAC}

The objective of load-based JCAC algorithms [2,12,13, $17,18]$ is to uniformly distribute traffic load among all the available RATs in a heterogeneous wireless network. A load-based JCAC algorithm will lead to unfairness in distribution of radio resources among heterogeneous mobile terminals.

\subsection{Path-Loss-Based JCAC}

Path-loss-based JCAC algorithms make call admission decisions based on path-loss measurements taken in the cells of each RAT. Perez-Romero et al. [2,14] have proposed path-loss based JCAC algorithms for new calls (initial RAT selection) and handoff calls (vertical handoff RAT selection) in heterogeneous CDMA/TDMA network. Path-loss-based JCAC algorithms can cause unfairness in distribution of radio resources among heterogeneous mobile terminals.

\subsection{Service-Cost-Based JCAC}

Service-cost-based JCAC algorithms [2,15] admit incoming calls into the least expensive RAT so that the subscriber incurs the lowest service cost in the heterogeneous wireless network. This approach is based on the fact that service cost differs from one RAT to another. Service-cost-based JCAC algorithms reduce the overall service cost incurred by subscribers. However, they can cause unfairness in distribution of radio resources among heterogeneous mobile terminals.

\subsection{Layer-Based JCAC}

In overlaid networks, layer-based JCAC algorithms admit calls based on the hierarchy of layers. If a layer cannot accommodate the call, the JCAC algorithm tries to admit the call in the next available layer. In [2,16], Ali and Pierre have proposed a layer-based predictive JCAC algorithm for overlaid heterogeneous wireless networks. Layer-based JCAC algorithms can lead to unfairness in distribution of radio resources among heterogeneous mobile terminals.

\subsection{RAT-Duplexing-Technique Based JCAC}

In this approach [19], incoming calls are admitted into a heterogeneous network based of RAT-duplexing techniques of the available RATs and service symmetry of the incoming calls. The main objective of the proposed algorithm is to reduce call blocking/dropping probability. The objective is achieved by admitting symmetry calls into non-flexible RATs and by admitting asymmetry calls into flexible RATs.

In the RAT selection approaches reviewed above, mobile terminal heterogeneity (modality) has not been considered in making RAT selection decisions. Consequently, there is a problem of unfairness in allocation of radio resources among heterogeneous mobile terminals. To solve the problem of unfairness discussed above, a terminal-modality-based JCAC scheme is proposed in Section 3.

\section{Proposed Terminal-Modality-Based JCAC Scheme}

This section describes the proposed terminal-modalitybased JCAC scheme which consists of the following two components: joint call admission controller and bandwidth reservation unit.

\subsection{The Joint Call Admission Controller}

The joint call admission controller implements the JCAC algorithm. The basic function of the JCAC algorithm is to make call admission decisions and guarantee fairness among the different heterogeneous terminals in the heterogeneous wireless network.

The proposed JCAC algorithm makes RAT selection decisions based on the modality of the mobile terminal initiating a call, the terminal support index of each RAT that can support the call, and the current load in the available RATs.

Terminal support index of a RAT indicates the ratio of terminals supported by the RAT to the total terminals registered in the heterogeneous wireless network. Terminal support index of RAT-j $\left(R^{j}\right)$ is defined as follows:

$$
R^{j}=\frac{T^{j}}{T}, 0<R^{j} \leq 1
$$

where $T^{j}$ is the number of mobile terminals that have an interface for RAT-j (i.e. that can be admitted into RAT-j), and $T$ is the total number of mobile terminals registered in the network.

During a call setup, a mobile terminal initiating a call sends a service request to the joint call admission controller which implements the JCAC algorithm. The JCAC algorithm is part of the joint resource management entity of the heterogeneous wireless network. The location of the joint resource management entity depends on the specific network architecture deployed by the network operator. The service request contains the call type, terminal-modality (number and types of network supported by the terminal), service class, and bandwidth 
requirements. Figure 2 is the flow chart of the proposed JCAC algorithm. As shown in Figure 2, $x_{i j}$, and $y_{i j}$ represent the residual bbu available for new and handoff class-i calls, respectively, in RAT-j. $L_{j}$, and $T^{j}$ represent the current load and Terminal support index of RAT-j, respectively. $H_{i}$ is the set of RATs that can support an incoming class-i call, and the mobile terminal (based on terminal modality) initiating the call. $h_{i}$ is the set of indexes of RATs that belong to set $H_{i}$.

As shown in Figure 2, whenever a class-i call arrives from a y-mode terminal in the heterogeneous wireless network, the JCAC algorithm determines the set $H_{i}$ of RAT-j that can support the class-i call and that can support the mobile terminal initiating the call (based on terminal modality). The JCAC algorithm then sorts the RATs in set $H_{i}$ in increasing order of their terminalsupport index $\left(R^{j}\right)$. Starting from the RAT with the lowest terminal-support index, the JCAC algorithm tries to admit the incoming class-i call into one of the RATs that can support the mobile terminal. If the RAT with the lowest terminal support index is fully loaded and therefore cannot accommodate the call, the next available RAT with the second-lowest terminal support index will be selected for the call, and so on. If two or more RATs that can support the incoming call have the same low terminal-support index, the least loaded of the two or more RATs will be selected for the incoming call.

The class-i call is rejected if none of the RATs in set $H_{i}$ has enough basic bandwidth units (bbu) to accommodate the call.

By attempting to admit an incoming call into the RAT with the lowest terminal-support index that can support the class of the call, the proposed TJCAC scheme reserves other RATs with a higher terminal-support index for calls from low-capability terminals, thereby reducing the blocking probability of such calls.

Figure 3 illustrates the problem of unfairness in radio resource allocation in a three-RAT heterogeneous wireless network when terminal modality is not considered in making RAT selection decisions. Assume that 1) all the three RATs have equal capacity; 2) all the arriving calls belong to the same class; and 3) each RAT can support only two calls. Figure 3 shows six consecutively arriving calls ( 1 to 6$)$ in the heterogeneous wireless network. A load-balancing JCAC scheme, for instance, will admit the first incoming call (call 1 from a triple-mode terminal) into RAT 1. It will admit call 2 (from a dual-mode terminal) into RAT 2, admit call 3 (from a triple-mode terminal) into RAT 3, and admit call 4 (from a dual-mode terminal into RAT 1). The JCAC scheme cannot admit the fifth incoming call (call 5) into RAT 2 or RAT 3 because call 5 is from a single-mode terminal that is supported only by RAT 1 . The JCAC scheme will then try to admit call 5 into RAT 1 . Call 5 will be blocked in RAT 1

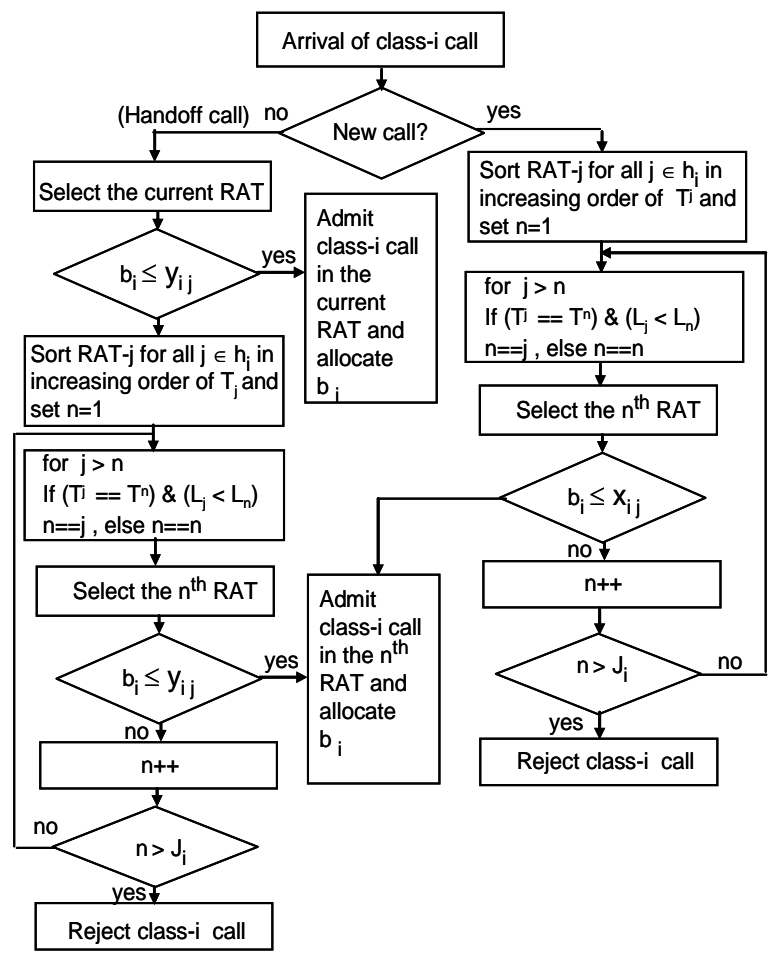

Figure 2. Flow chart of the proposed TJCAC algorithm.

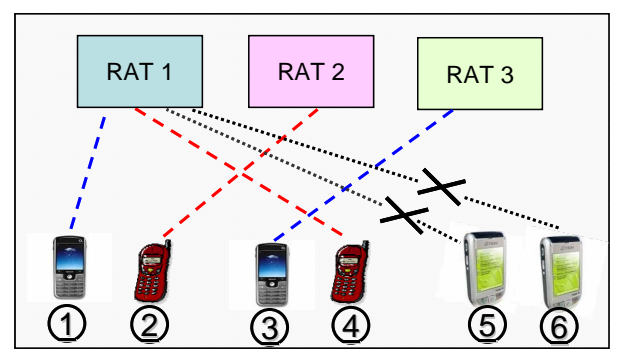

Figure 3. Unfair allocation of radio resources among heterogeneous mobile terminals.

because it is already fully loaded (maximum of two calls). In a similar manner, the sixth incoming call (call 6) from a single-mode terminal that is supported by RAT 1 only, will be blocked.

Figure 4 illustrates how the proposed JCAC scheme reduces the problem of unfairness in allocation of radio resources among heterogeneous mobile terminals. As show in Figure 4, and using Equation (1), the terminal support index of RAT 1, RAT 2, and RAT 3 are 6/6, 4/6, and 2/6 respectively. Note that terminal support index is calculated based on the number of terminals registered in the heterogeneous wireless network, and it is assumed that there only six terminals in this example.

As shown in Figure 4, the first incoming call (call 1 from a triple-mode terminal) can be admitted into any of the three RATs. The proposed TJCAC will select the RAT with the lowest terminal support index (RAT 3), thereby reserving RAT 1 and RAT 2 for calls from 


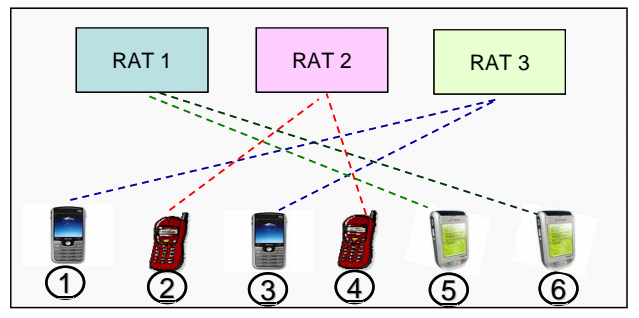

Figure 4. Fair allocation of radio resources among heterogeneous mobile terminals.

low-capability mobile terminals. The second incoming call (call 2 from a dual-mode terminal) can be admitted into RAT 1 or RAT 2, The TJCAC scheme selects RAT 2 , which has the lower terminal support index. The third incoming call (call 3 is from a triple mode terminal) can be admitted into any of the three RATs. The TJCAC selects RAT 3 with the lowest terminal support index. The fourth call is admitted into RAT 2. The fifth and sixth calls are then admitted into RAT 1, which has the highest terminal support index. Thus the problem of unfairness in allocation of radio resources is reduced.

\subsection{Bandwidth Allocation Unit}

In order to maintain lower handoff dropping probability over new call blocking probability, different thresholds are used for rejecting new and handoff calls. In RAT-j, $C_{j}$ and $t_{0 j}$ are the thresholds for rejecting handoff calls and new calls respectively. Figure 5 shows the different thresholds used by the bandwidth allocation unit.

\section{Heterogeneous Network Model}

This paper considers a heterogeneous cellular network which comprises a set of RATs $H$ with co-located cells supporting heterogeneous terminals. A typical example of a heterogeneous wireless network, adapted from [20] is shown in Figure 6.

In the heterogeneous network, radio resources are jointly managed. Cellular networks such as GSM, UMTS, EV-DO can have the same and fully overlapped coverage, which is technically feasible, and may also save on installation costs [11,21].

Let $H$ denote the set of all available RATs in the heterogeneous wireless network. Then, $H$ is given as follows:

$$
H=\{R A T 1, R A T 2, \cdots, R A T j\}
$$

where $j$ is the total number of RATs in the heterogeneous cellular network. The heterogeneous cellular network supports k-classes of calls.

Each cell in RAT-j $(j=1, \cdots, J)$ has a total of $C_{j}$ basic bandwidth units (bbu). The physical meaning of a unit of radio resources (such as time slots, code sequence, etc.) is dependent on the specific technological implementation of the radio interface [22]. However, no matter which

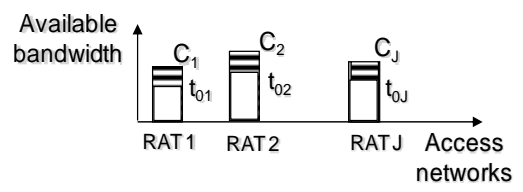

Figure 5. Bandwidth allocation policy.

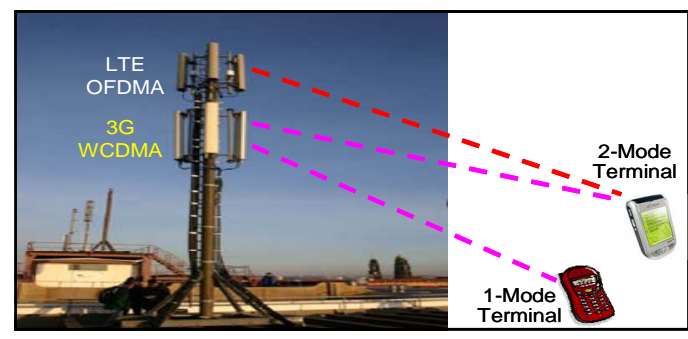

Figure 6. A typical two-RAT heterogeneous cellular network with co-located cells.

multiple access technology (FDMA, TDMA, CDMA or OFDMA) is used, system capacity could be interpreted in terms of effective or equivalent bandwidth [23-25]. Therefore, this paper refers to the bandwidth of a call as the number of bbu that is adequate for guaranteeing the desired QoS for the call, which is similar to the approach used for homogeneous networks in $[25,26]$.

The approach used is to decompose a heterogeneous cellular network into groups of co-located cells as shown in Figure 7. For example, cell 1a and cell 2a form a group of co-located cells. Similarly, cell $1 \mathrm{~b}$ and cell $2 \mathrm{~b}$ form another group of co-located cells, and so on.

When a mobile user with an ongoing call is moving outside the coverage area of a group of co-located cells, the call must be handed over to one of the cells that can support the call in the neighboring group of co-located cells. For example, in the two-class three-RAT heterogeneous cellular network illustrated in Figure 7, an ongoing class-1 call can be handed over from cell 2a to cell $2 \mathrm{~b}$, or from cell 2a to cell 1b (Figure 7). Note that handoff comprises both horizontal and vertical handoffs.

The correlation between the groups of co-located cells results from handoff connections between the cells of corresponding groups. Under this formulation, each group of co-located cells can be modeled and analyzed individually. Therefore, a single group of co-located cells is considered in this paper.

The heterogeneous network supports k classes of calls. Each class is characterized by bandwidth requirement, arrival distribution, and channel holding time. Each classi call requires a discrete bandwidth value, $b_{i}$. Following the general assumption in cellular networks, new and handoff class-i calls arrive in the group of co-located cells according to Poisson process with rate $\lambda_{i}^{n}$ and $\lambda_{i}^{h}$ respectively. The call holding time (CHT) of a class-i call is assumed to follow an exponential distribution with mean $1 / \mu_{c i}[25,27]$. 


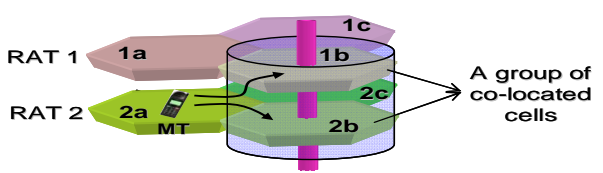

Figure 7. A two-RAT heterogeneous wireless network supporting heterogeneous mobile terminals.

The cell residence time (CRT) is assumed to follow an exponential distribution with mean $1 / \mu_{h i}$. The channel holding time for class-i calls, is exponentially distributed with mean $1 / \mu_{i}$ where $\mu_{i}=\left(\mu_{c i}+\mu_{h i}\right)$ [27]. Note that this set of assumptions has been widely used for both homogeneous and heterogeneous cellular networks in the literature, and is found to be generally applicable in the networks where the number of mobile users is larger than the number of channels.

\section{Heterogeneous Terminal Model}

Heterogeneous mobile terminals ranging from singlemode terminals to J-mode terminals are considered in this paper, where $\mathrm{J}$ is the total number of RATs in the heterogeneous wireless network. The heterogeneous terminals can be broadly categorized into J groups based on the number of RATs supported by each terminal. For example, in a three-RAT heterogeneous wireless network, there will be a maximum of three categories of mobile terminals namely: 1-mode (single-mode), 2-mode (dualmode), and 3-mode (triple-mode) terminals. This categorization is based on the number of usable network interfaces possessed by each terminal in the heterogeneous network.

For instance, a 4-mode (quad-mode) terminal in a three-RAT heterogeneous wireless network will have a maximum of three usable interfaces in the heterogeneous network because there is a maximum of three RATs available. Therefore, the 4-mode terminal will be categorized as a 3-mode terminal in a three-RAT heterogeneous network provided all the three-RATs are supported by the terminal. The foregoing implies that in a J-RAT heterogeneous wireless network, a subscriber's terminal can only have a maximum of $\mathrm{J}$ usable interfaces (J-mode terminals).

In a J-RAT heterogeneous wireless network, the y-mode terminals $(y \in[1, J])$ can be subdivided into $T_{y}^{J}$ types. Let $M t^{y, x}$ signify a y-mode terminal $(y \in[1, J])$ of type $\mathrm{x}\left(x \in\left[1, T_{y}^{J}\right]\right.$, and $T_{y}^{J}$ is the maximum number of types of a y-mode terminal in a J-RAT heterogeneous network.

For a y-mode terminal $T_{y}^{J}$ is obtained as follows:

$$
T_{y}^{J}={ }^{J} C_{y}
$$

where ${ }^{J} C_{y}$ represents $J$ combination $y$.

For example, in a three-RAT heterogeneous wireless network, the maximum number of types of 1-mode terminals is calculated as:

$$
T_{1}^{3}={ }^{3} C_{1}=3
$$

Similarly, the number of types of 2-mode terminals are $T_{2}^{3}={ }^{3} C_{2}=3$, and the maximum number of types of 3-mode terminals is $T_{3}^{3}={ }^{3} C_{3}=1$.

The classification of heterogeneous mobile terminals in a three-RAT heterogeneous wireless network is illustrated in Figures 8-10.

As shown in Figure 8, there are three types of 1-mode terminals in the three-RAT network. The first type of mobile terminals $\left(M t^{1,1}\right)$ has a single network interface that can only support RAT-1. The second $\left(M t^{1,2}\right)$ and third $\left(M t^{1,3}\right)$ types of 1-mode terminals also have a single network interface that can support RAT 2 and RAT 3, respectively.

In Figure 9, there are three possible types of 2-mode terminals. The first type of 2-mode terminals $\left(M t^{2,1}\right)$ has two network interfaces that can support RAT-1 and RAT-2, one interface for each RAT. Similarly, the second type of 2-mode terminals $\left(M t^{2,2}\right)$ has two network interfaces that can support RAT-1 and RAT-3, one interface for each RAT. The third type of two-mode terminals

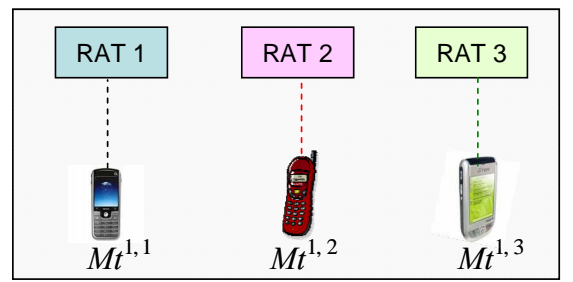

Figure 8. Classification of 1-mode terminals in a three-RAT heterogeneous wireless network.

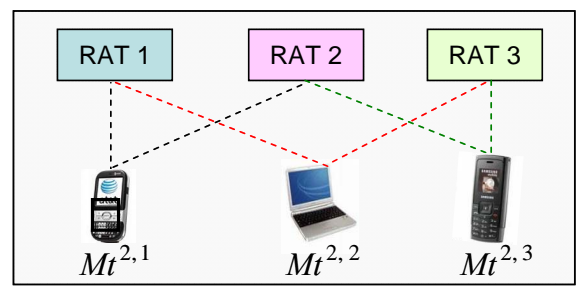

Figure 9. Classification of 2-mode terminals in a three-RAT heterogeneous wireless network.

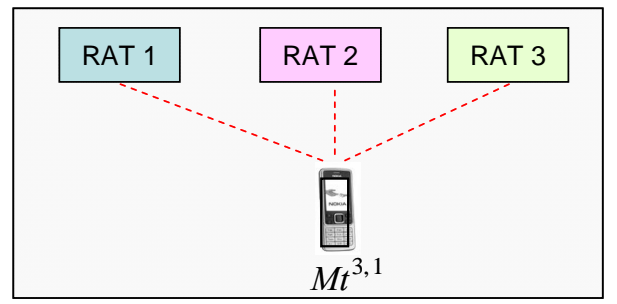

Figure 10. Classification of 3-mode terminals in a threeRAT heterogeneous wireless network. 
$\left(M t^{2,3}\right)$ has two network interfaces that can support RAT-2 and RAT-3, one interface for each RAT.

Figure 10 shows the only possible type of 3-mode terminals in the three-RAT heterogeneous wireless network. The 3-mode terminals have three usable network interfaces, one for each RAT.

The total number of types of heterogeneous mobile terminals $\left(T^{J}\right)$ in a J-RAT heterogeneous wireless network is obtained as:

$$
T^{J}=\sum_{y=1}^{J}{ }^{J} C_{y}
$$

For a three-RAT heterogeneous wireless network, $T^{3}=7$.

In a J-RAT heterogeneous wireless network, let $P s_{y}^{j}$ be the probability that a y-mode terminal supports RAT-j (RAT-j $\in H$ ) and let $P s^{j}$ be the probability that any mobile terminal supports RAT-j. Then $P s_{y}^{j}$ is obtained as follows:

$$
P s_{y}^{j}=\frac{{ }^{J-1} C_{y-1}}{{ }^{J} C_{y}}=\frac{y}{J}
$$

$P s^{j}$ is obtained as:

$$
P s^{j}=\sum_{y=1}^{J} R_{y} \frac{y}{J}
$$

where $R_{y}$ is the percentage of subscribers using y-mode terminals.

In a J-RAT heterogeneous wireless network, let $P^{y, x}$ be the probability that a $y$-mode terminal $(y \in[1, J])$ is of type-x $\left(x \in\left[1, T_{y}^{J}\right]\right)$. Moreover, let $P b_{i}^{j}$ be the probability that a new class-i call from any terminal is blocked in RAT-j in the heterogeneous network. Let $d^{y, x}$ be the set of indices all RATs supported by a $\mathrm{y}$-mode terminal of type $\mathrm{x}$.

Then the probability, $P b_{y, i}$ that a new class-i call from a y-mode terminal is blocked in the heterogeneous wireless network is given as:

$$
P b_{y, i}=\sum_{x=1}^{T_{y}^{J}} \prod_{j \in d^{y, x}} P^{y, x} \cdot P b_{i}^{j}
$$

The probability, $P b_{i}$ that a new class-i call from any terminal is blocked in the heterogeneous wireless network is given as:

$$
P b_{i}=\sum_{y=1}^{J} P b_{y, i} \cdot R_{y}
$$

Similarly, the probability, $P d_{y, i}$ that a new class-i call from a y-mode terminal is dropped in the heterogeneous wireless network is given as:

$$
P d_{y, i}=\sum_{x=1}^{T_{y}^{J}} \prod_{j \in d^{y, x}} P^{y, x} \cdot P d_{i}^{j}
$$

The probability, $P d_{i}$ that a handoff class-i call from any terminal is dropped in the heterogeneous wireless network is given as:

$$
P d_{i}=\sum_{y=1}^{J} P d_{y, i} R_{y}
$$

In Section 6, expressions for $P b_{i}^{j}$ and $P d_{i}^{j}$ are derived.

\section{Markov Model}

The TJCAC and bandwidth management scheme described in Section 2 can be modeled as a multidimensional Markov chain. The current state of the heterogeneous system is represented as follows:

$$
\Omega=\left(m_{i, j}, n_{i, j}: i=1, \cdots, k, j=1, \cdots, J\right)
$$

The non-negative integer $m_{i, j}$ denotes the number of ongoing new class-i calls in RAT-j, and the non-negative integer $n_{i, j}$ denotes the number of ongoing handoff class-i calls in RAT-j.

Let $S$ denote the state space of all admissible states of the group of co-located cells as it evolves over time. An admissible state $\boldsymbol{s}$ is a combination of the numbers of users in each class that can be supported simultaneously in the group of co-located cells while maintaining adequate QoS and meeting resource constraints.

Let $S$ denote the state space of all admissible state as it evolves over time. An admissible state $s$ is a combination of the numbers of users in each class that can be simultaneously supported in the group of co-located cells while maintaining adequate QoS and meeting resource constraints. The state $S$ of all admissible states is given as:

$$
\begin{aligned}
\mathrm{S}=\{ & \left\{=\left(m_{i, j}, n_{i, j}: i=1, \cdots, k, j=1, \cdots, J\right):\right. \\
& \left.\sum_{i=1}^{k} m_{i, j} b_{i} \leq t_{0, j} \forall j \wedge \sum_{i=1}^{k}\left(m_{i, j}+n_{i, j}\right) b_{i} \leq C_{j} \forall j\right\}
\end{aligned}
$$

where $t_{0 j}$ and $C_{j}$ are thresholds for rejecting new and handoff class-i calls respectively, in RAT-j, and $b_{i}$ is the bbu allocated to an incoming class-i call in heterogeneous wireless network.

The constraints simply state that the sum of the bandwidth units of all admitted class-i calls cannot be more than the total bandwidth units available for that class of calls.

The call admission decision epochs are the arrival of a new or handoff call. When the system is in state $\boldsymbol{s}$, an accept/reject decision must be made for each type of possible arrival in the group of co-located cells. The possible JCAC decisions in the arrival epoch are reject the new (or handoff) class-i call or admit the new or handoff class-i call into RAT-j (RAT-j $\in H_{i}$ ), in which case the 
state $\boldsymbol{s}$ evolves.

Thus, the call admission action space A can be expressed as follows:

$$
\begin{aligned}
A=\{ & \left\{a=\left(a_{i}^{n}, \cdots, a_{k}^{n}, a_{i}^{h}, \cdots, a_{k}^{h}\right):\right. \\
& \left.a_{i}^{n}, a_{i}^{h} \in(0,1, \cdots, J), i=1, \cdots, k\right\}
\end{aligned}
$$

where $a_{i}^{n}$ denotes the action taken on arrival of a new class-i call within the group of co-located cells, and $a_{i}^{h}$ denotes the action taken on arrival of a handoff class-i call from an adjacent group of co-located cells. $a_{i}^{n}$ (or $\left.a_{i}^{h}\right)=0$ means reject the new (or handoff) class-i call. $a_{i}^{n} \quad$ (or $\left.a_{i}^{h}\right)=j$ means accept the new (or handoff) class-i call into RAT-j $(j \in H)$.

When a new or handoff class-i call arrives from a y-mode terminal into a group of co-located cells, the JCAC algorithm selects a RAT in set $H_{i}$ for the incoming call, where $H_{i}$ is the set of RATs supported by the $\mathrm{y}$-mode terminal. The action of selecting a RAT for each arriving new or handoff class-i call in the group of co-located cells leads to splitting of the arrival process.

Let $\lambda_{i}^{n}$ and $\lambda_{i}^{h}$ denote the mean arrival rates of new class-i calls and handoff class-i calls, respectively, in the group of co-located cells. Furthermore, let $\lambda_{i j}^{n}$ and $\lambda_{i j}^{h}$ denote the mean arrival rates of new class-i calls and handoff class-i calls, respectively in RAT-j.

The arrival rate of class-i calls in the group of colocated cells is split among all the RATs in set H. Each RAT has a fraction of the arrival rate $\left(\lambda_{i}^{n}\right)$.

Let $\alpha_{i j}$ and $\beta_{i j}$ denote the fraction of new and handoff class-i calls admitted into RAT-j respectively (RAT-j $\in$ $H$ ). The arrival rate of new class-i calls into RAT-j is given as:

$$
\lambda_{i, j}^{n}=\alpha_{i, j} \lambda_{i}^{n} \quad i=1, \cdots, k, \quad j=1, \cdots, J
$$

Similarly, the arrival rate of handoff class-i calls into RAT-j is given as:

$$
\lambda_{i, j}^{h}=\beta_{i, j} \lambda_{i}^{n} \quad i=1, \cdots, k, \quad j=1, \cdots, J
$$

The total arrival rate of new and handoff calls into RAT-j is given as:

$$
\lambda_{j}^{T}=\sum_{i=1}^{K}\left(\alpha_{i, j} \lambda_{i, j}^{n}+\beta_{i, j} \lambda_{i, j}^{h}\right) \forall j
$$

Note that the arrival rates of a split Poisson process are also Poisson [28]. Therefore, given that the mean arrival rate of class-i calls into the group of co-located cells is Poisson, the mean arrival rates of the split class-i calls into RAT-j ( $\forall$ RAT $j \in H$ ) are also Poisson.

From the steady state solution of the Markov model, performance measures of interest can be determined by summing up appropriate state probabilities. Let $P(s)$ denote the steady state probability that the system is in state $s(s \in S)$. From the detailed balance equation, $P(s)$ is obtained as:

Let $\rho_{\text {eve }_{i, j}}$ and $\rho_{\text {han }_{i, j}}$ denote the load generated by new class-i calls and handoff class-i calls, respectively, in RAT-j. Then,

$$
\rho_{\text {new }_{i, j}}=\frac{\lambda_{i, j}^{n}}{\mu_{i}^{n}} \quad \forall i, j,
$$

and

$$
\rho_{h a n_{i, j}}=\frac{\lambda_{i, j}^{h}}{\mu_{i}^{h}} \quad \forall i, j
$$

From the steady state solution of the Markov model, performance measures of interest can be determined by summing up appropriate state probabilities. Let $P(s)$ denote the steady state probability that the group of collocated cells in the heterogeneous wireless network is in state $\boldsymbol{s}(\boldsymbol{s} \in \boldsymbol{S})$. From the detailed balance equation, $P(s)$ is obtained as:

$$
P(s)=\frac{1}{G} \prod_{i=1}^{k} \prod_{j=1}^{J} \frac{\left(\rho_{\text {new }_{i, j}}\right)^{m_{i, j}}}{m_{i, j} !} \frac{\left(\rho_{\text {han }_{i, j}}\right)^{n_{i, j}}}{n_{i, j} !} \forall s \in S
$$

where $G$ is a normalization constant given by:

$$
G=\sum_{s \in S} \prod_{i=1}^{k} \prod_{j=1}^{J} \frac{\left(\rho_{\text {nev }_{i, j}}\right)^{m_{i, j}}}{m_{i, j} !} \frac{\left(\rho_{\text {han }_{i, j}}\right)^{n_{i, j}}}{n_{i, j} !}
$$

\subsection{New Call Blocking Probability}

A new class-i call from a y-mode terminal is blocked in the group of co-located cells if none of the available RATs supported by the y-mode terminal has enough bbu to accommodate the new class-i call. Let $S_{b i}^{j} \subset S$ denote the set of states in which a new class-i call is blocked in RAT-j in the group of co-located cells. It follows that:

$$
\begin{aligned}
S b_{i}^{j}=\left\{s \in S:\left(b_{i}+\sum_{x=1}^{k} m_{x, j} b_{x}>t_{0 j} \vee\right.\right. \\
\left.\left.b_{i}+\sum_{x=1}^{k}\left(m_{x, j}+n_{x, j}\right) b_{x}>C_{j}\right)\right\}
\end{aligned}
$$

Thus the new blocking probability (NCBP), $P b_{i}^{j}$ for a class-i call in RAT-j in the group of co-located cell is given by:

$$
P b_{i}^{j}=\sum_{s \in S b_{i}^{j}} P(s)
$$

\subsection{Handoff Call Dropping Probability}

A handoff class-i call from a y-mode terminal is dropped in the group of co-located cells if none of the available RATs supported by the y-mode terminal has enough bbu to accommodate the handoff class-i call. Let $S d_{i}^{j} \subset S$ denote the set of states in which a handoff class-i call is dropped in the group of co-located cells. It follows that: 


$$
S d_{i}^{j}=\left\{s \in S:\left(b_{i}+\sum_{x=1}^{k}\left(m_{x, j}+n_{x, j}\right) b_{x}>C_{j}\right)\right\}
$$

Thus the handoff dropping probability (HCDP), $P d_{i}^{j}$, for a class-i call in RAT-j in the group of co-located cell is given by:

$$
P d_{i}^{j}=\sum_{s \in S d_{i}^{j}} P(s)
$$

\section{Results}

In this section, the performance of the proposed terminal-modality based JCAC scheme is evaluated with respect to NCBP and HCDP, using a two-class threeRAT heterogeneous wireless network supporting heterogeneous mobile terminals. A numerical simulation is conducted using MATLAB. The following system parameters are used: $C_{1}=C_{2}=C_{3}=10, t_{01}=t_{02}=t_{03}=5$, $b 1=1, b 2=3, \mu_{1}=\mu_{2}=0.5, \lambda_{1}^{n}=[1,5], \lambda_{2}^{n}=\lambda_{1}^{n}$, $\lambda_{i}^{h}=0.5 \lambda_{i}^{n}$.

Different combinations of heterogeneous terminals are possible in heterogeneous cellular networks. However, in order to investigate the performance of the proposed JCAC scheme, four scenarios with different combinations of heterogeneous terminals are examined.

For each of the four scenarios, the performance of the proposed TJCAC scheme (represented as "T" scheme) is compared with the performance of two other JCAC schemes namely, the equal-probability selection-based JCAC scheme (represented as "E" scheme) and the service-class-based JCAC scheme (represented as " $\mathrm{S}$ " scheme). Results obtained from the four scenarios are discussed in the following subsections. A preliminary of these results has been presented in [29].

\subsection{Scenario 1: Equal Ratio of Heterogeneous Terminals}

In this scenario, the ratio of single-mode, dual-mode, and triple-mode terminals is 33:33:33, respectively. Figure $\mathbf{1 1}$ shows the effect of varying the new call arrival rate on the NCBP $(\mathrm{Pb} 1)$ of 1-mode, 2-mode, and 3-mode terminals for EJCAC, SJCAC, and the proposed TJCAC. As shown in Figure 11, Pb1 for the three JCAC schemes and three classes of terminals increases with an increase in arrival rate for three JCAC schemes. This is expected. However, for 1-mode terminals, $\mathrm{Pb} 1$ of the TJCAC scheme is lower than the corresponding Pb1 of the EJCAC and SJCAC schemes. The TJCAC scheme is able to reduce the Pb1 by admitting most of the calls from 3-mode terminals into RAT 3, which has the lowest terminal support index, thereby reserving RAT 1 and RAT 2 for calls from 1-mode and 2-mode terminals, respectively. However, the reduction in 1-mode terminals $\mathrm{Pb} 1$ for TJCAC scheme is at the expense of the Pb1 of calls from

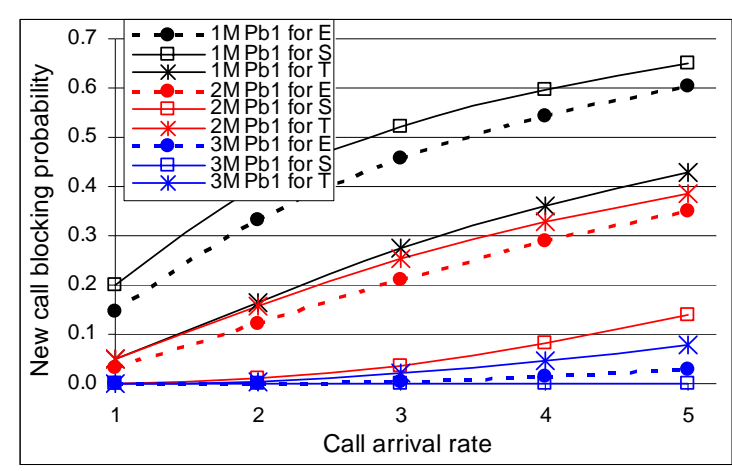

Figure 11. NCBP (Pb1) for class-1 calls with 1 M:2 M:3 M = 33:33:33.

3-mode terminals. It can be seen that for 3-mode terminals, the $\mathrm{Pb} 1$ of the TJCAC scheme is a little higher than the corresponding $\mathrm{Pb} 1$ of the EJCAC and SJCAC schemes.

For the EJCAC scheme, the Pb1 for one-mode terminals (1 M Pb1) is very high whereas $\mathrm{Pb} 1$ for two-mode and three-mode terminals are relatively low. Thus, onemode terminals are treated unfairly by the EJCAC scheme. Similarly, for SJCAC the Pb1 for one-mode terminals $(1 \mathrm{M} \mathrm{Pb} 1)$ is very high whereas $\mathrm{Pb} 1$ for two-mode and three-mode terminals are very low. Thus, one-mode terminals are treated unfairly by the SJCAC scheme.

However, for the proposed TJCAC scheme, Pb1 for one-mode terminals is relatively low, thereby reducing unfairness in allocation of radio resources among the heterogeneous terminals.

Figure 12 shows the effect of varying the new call arrival rate on the NCBP $(\mathrm{Pb} 2)$ of 1-mode, 2-mode, and 3-mode terminals for EJCAC, SJCAC, and the proposed TJCAC. The Pb2 for the three JCAC schemes follow a similar trend to that of Figure 11. The $\mathrm{Pb} 2$ for the three JCAC schemes and three classes of terminals increases with an increase in arrival rate for three JCAC schemes. This is expected. However, for the EJCAC scheme, the $\mathrm{Pb} 2$ for one-mode terminals $(1 \mathrm{M} \mathrm{Pb} 2)$ is very high whereas the $\mathrm{Pb} 2$ for two-mode and three-mode terminals are relatively low. Thus, one-mode terminals are treated unfairly by the EJCAC scheme. Similarly, for the SJCAC the $\mathrm{Pb} 2$ for one-mode terminals $(1 \mathrm{M} \mathrm{Pb} 2)$ is very high whereas $\mathrm{Pb} 2$ for two-mode and three-mode terminals are very low. Thus, one-mode terminals are treated unfairly by the SJCAC scheme.

However, for the proposed TJCAC scheme, the $\mathrm{Pb2}$ for one-mode terminals is relatively low, thereby reducing unfairness in allocation of radio resources among the heterogeneous terminals.

Figure 13 shows the effect of varying the call arrival rate on the HCDP (Pd1) of 1-mode, 2-mode, and 3-mode terminals for EJCAC, SJCAC, and the proposed TJCAC. Pd1 for the three JCAC schemes follow a similar trend to 


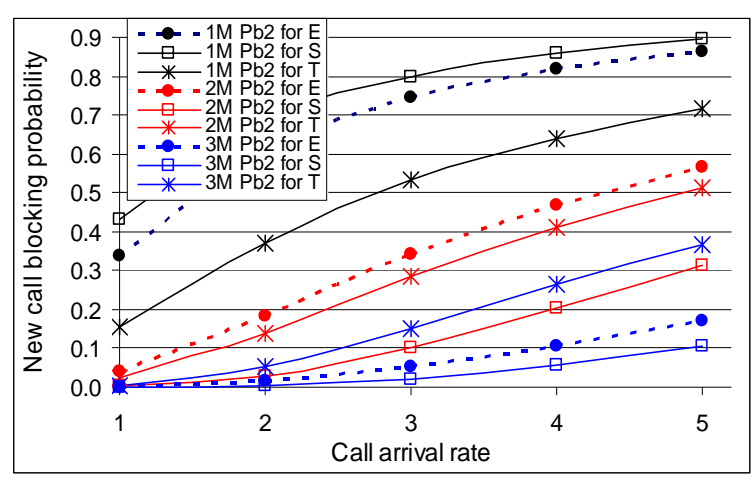

Figure 12. NCBP (Pb2) for class-2 calls with $1 \mathrm{M}: 2 \mathrm{M}: 3 \mathrm{M}=$ 33:33:33.

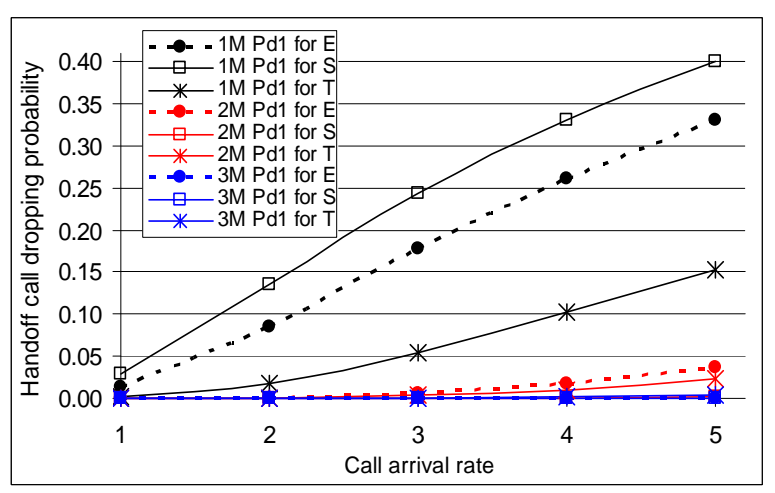

Figure 13. HCDP (Pd1) for class-1 calls with 1 M:2 M:3 M $=33: 33: 33$.

that of Figure 11. Pd1 for the three JCAC schemes and three classes of terminals increases with an increase in arrival rate for three JCAC schemes. This is expected. However, For the EJCAC scheme, the Pd1 for one-mode terminals (1 M Pd1) is very high whereas Pd1 for twomode and three-mode terminals is relatively low. Thus, one-mode terminals are treated unfairly by the EJCAC scheme. Similarly, for SJCAC the Pd1 for one-mode terminals (1 M Pd1) is very high whereas Pd1 for twomode and three-mode terminals are very low. Thus, onemode terminals are treated unfairly by the SJCAC scheme.

However, for the proposed TJCAC scheme, the Pd1 for one-mode terminals is relatively low, thereby reducing unfairness in allocation of radio resources among the heterogeneous terminals.

Figure 14 shows the effect of varying the call arrival rate on the HCDP (Pd2) of 1-mode, 2-mode, and 3-mode terminals for EJCAC, SJCAC, and the proposed TJCAC. The Pd2 for the three JCAC schemes follow a similar trend to that of Figure 11. Pd2 for the three JCAC schemes and three classes of terminals increases with an increase in arrival rate for three JCAC schemes. This is expected. However, For the EJCAC scheme, the Pd2 for one-mode terminals (1 M Pd2) is very high whereas the Pd1 for two-mode and three-mode terminals are relatively

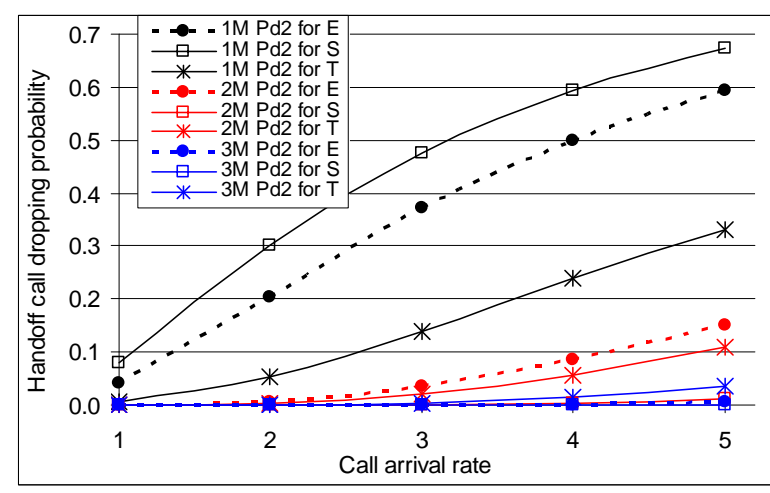

Figure 14. HCDP (Pd2) for class-2 calls with 1 M:2 M:3 M $=33: 33: 33$.

low. Thus, one-mode terminals are treated unfairly by EJCAC scheme. Similarly, for the SJCAC the Pd2 for one-mode terminals (1 M Pd2) is very high whereas the Pd2 for two-mode and three-mode terminals are very low. Thus, one-mode terminals are treated unfairly by SJCAC scheme.

However, for the proposed TJCAC scheme, the Pd2 for one-mode terminals is relatively low, thereby reducing unfairness in allocation of radio resources among the heterogeneous terminals.

\subsection{Scenario 2: Terminals Dominated by 1-Mode Terminals}

In this scenario, the ratio of single-mode, dual-mode, and triple-mode terminals is 50:25:25 respectively. Figures 15, 16, 17, and 18 show Pb1, Pb2, Pd1, and Pd2 respectively. It can be seen that the proposed TJCAC reduces the $\mathrm{Pb} 1, \mathrm{~Pb} 2$, Pd1, and Pd2 of 1-mode terminals, in Figures $15,16,17$, and 18 , respectively, thereby reducing unfairness in allocation of radio resources among heterogeneous wireless terminals in the heterogeneous wireless network.

\subsection{Scenario 3: Terminals Dominated by 2-Mode Terminals}

In this scenario, the ratio of single-mode, dual-mode, and triple-mode terminals is 25: 50: 25 respectively. Figures 19, 20, 21, and 22 show $\mathrm{Pb} 1, \mathrm{~Pb} 2$, Pd1, and Pd2 respectively. It can be seen that the proposed TJCAC reduces the Pb1, Pb2, Pd1, and Pd2 of 1-mode terminals, in Figures $19,20,21$, and 22 , respectively, thereby reducing unfairness in allocation of radio resources among heterogeneous wireless terminals in the heterogeneous wireless network.

\subsection{Scenario 4: Terminals Dominated by 3-Mode Terminals}

In this scenario, the ratio of single-mode, dual-mode, and 


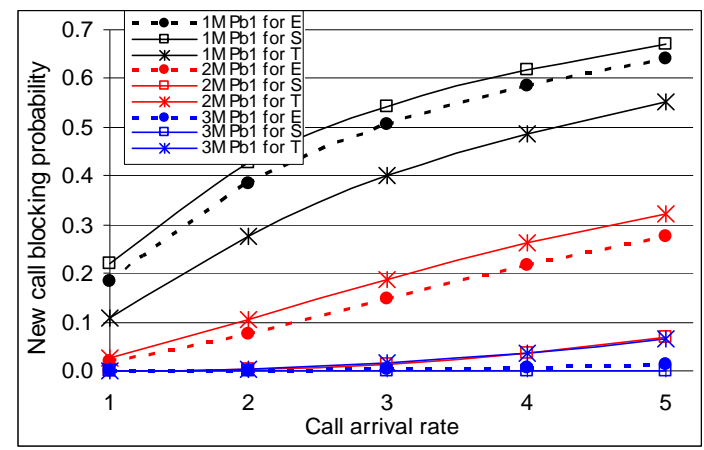

Figure 15. NCBP (Pb1) for class-1 calls with 1 M:2 M:3 $M=$ 50:25:25.

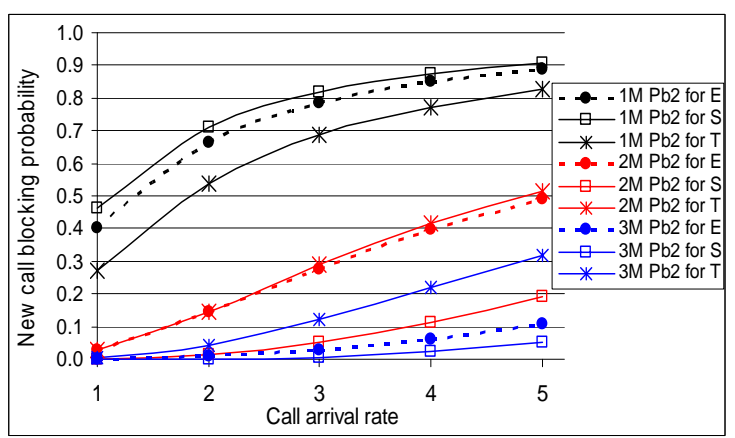

Figure 16. NCBP (Pb2) for class-2 calls with $1 \mathrm{M}: 2 \mathrm{M}: 3 \mathrm{M}=$ 50:25:25.

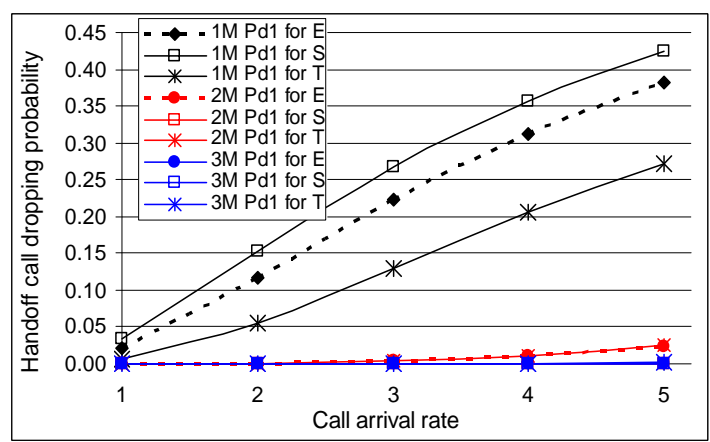

Figure 17. HCDP (Pd1) for class-1 calls with 1 M:2 M:3 M $=50: 25: 25$.

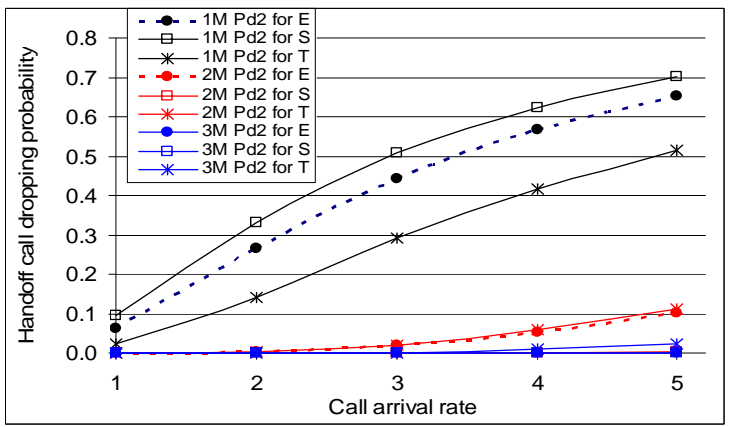

Figure 18. HCDP (Pd2) for class-2 calls with 1 M:2 M:3 M $=50: 25: 25$.

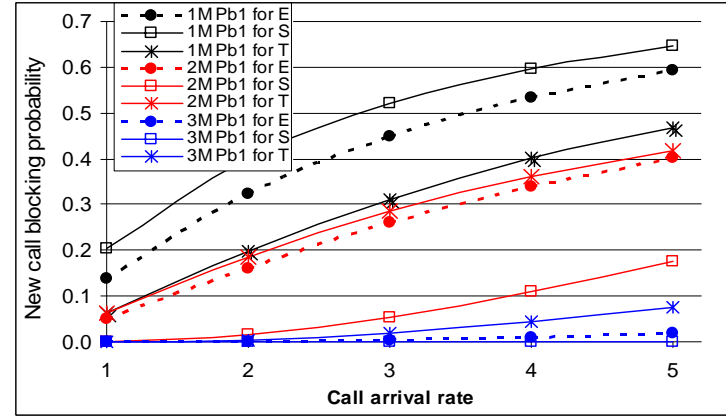

Figure 19. NCBP (Pb1) for class-1 calls with 1 M:2 M:3 $M=$ 25:50:25

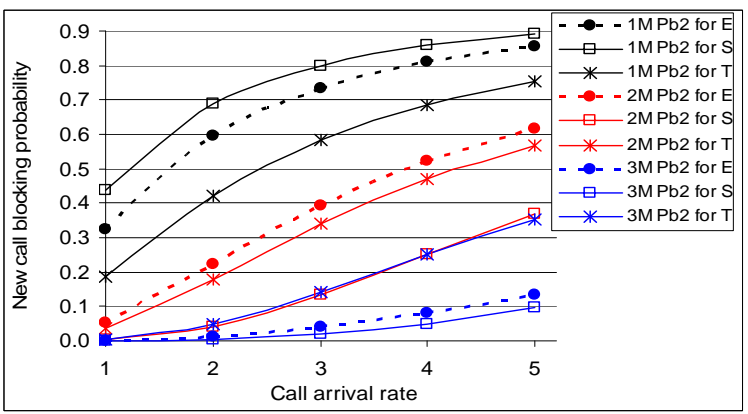

Figure 20. NCBP (Pb2) for class-2 calls with 1 M:2 M:3 M = 25:50:25.

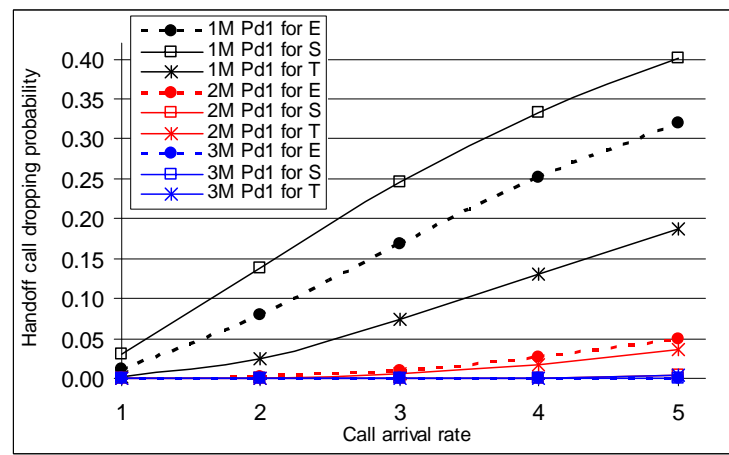

Figure 21. HCDP (Pd1) for class-1 calls with 1 M:2 M:3 M $=25: 50: 25$.

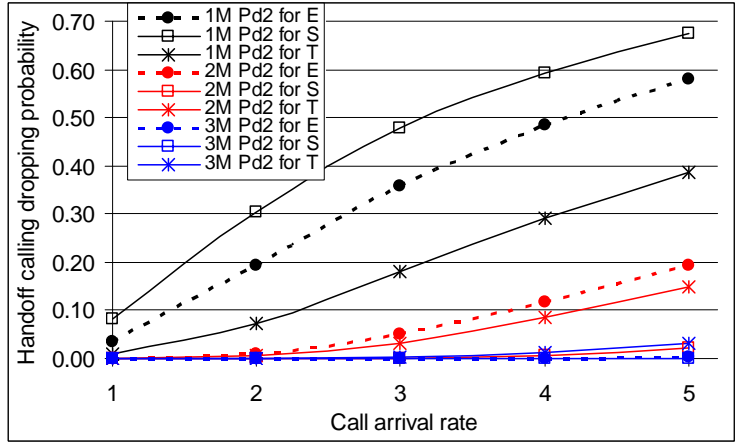

Figure 22. HCDP (Pd2) for class-2 calls with 1 M:2 M:3 M $=25: 50: 25$. 
triple-mode terminals is 25:25:50 respectively. Figures 23, 24, 25, and 26 show Pb1, Pb2, Pd1, and Pd2 respectively. It can be seen that the proposed TJCAC reduces $\mathrm{Pb} 1, \mathrm{~Pb} 2, \mathrm{Pd} 1$, and $\mathrm{Pd} 2$ of 1-mode terminals, in Figures $23,24,25$, and 26, respectively, thereby reducing unfairness in allocation of radio resources among heterogeneous wireless terminals in the heterogeneous wireless network.

\section{Conclusion}

A terminal-modality-based JCAC scheme has been proposed for heterogeneous wireless network supporting

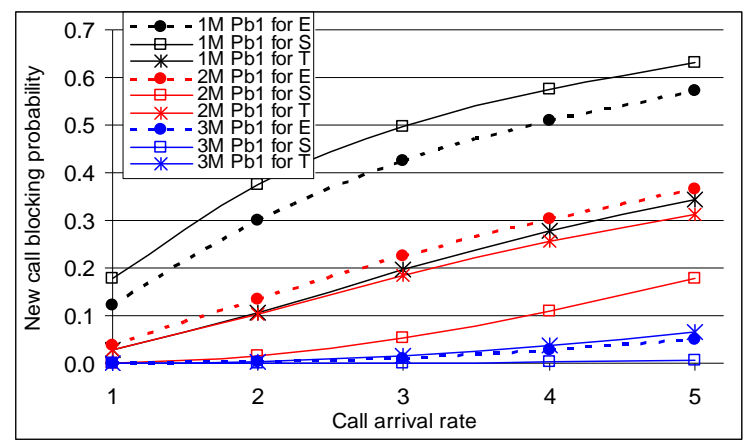

Figure 23. NCBP (Pb1) for class-1 calls with 1 M:2 M:3 $M=$ 25:25:50.

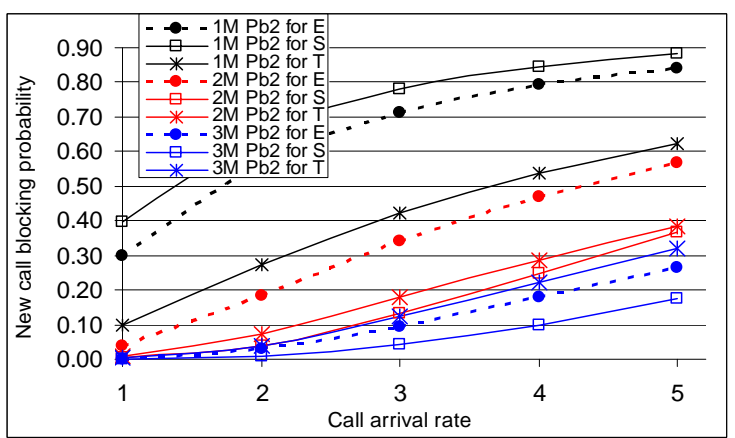

Figure 24. NCBP (Pb2) for class-2 calls with 1 M:2 M:3 M = 25:25:50.

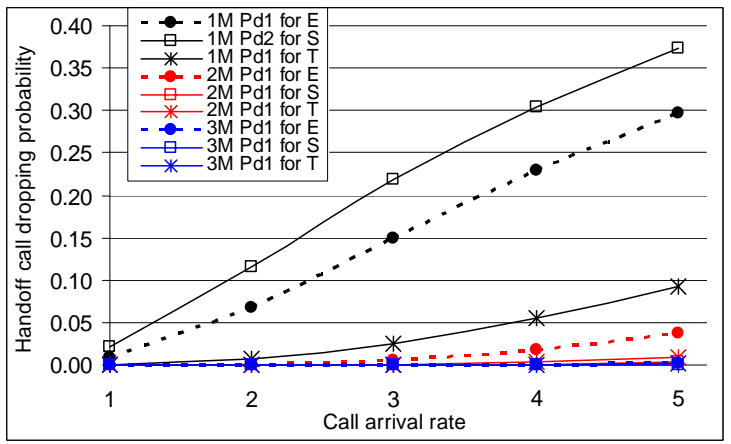

Figure 25. HCDP (Pd1) for class-1 calls with 1 M:2 M:3 M $=25: 25: 50$.

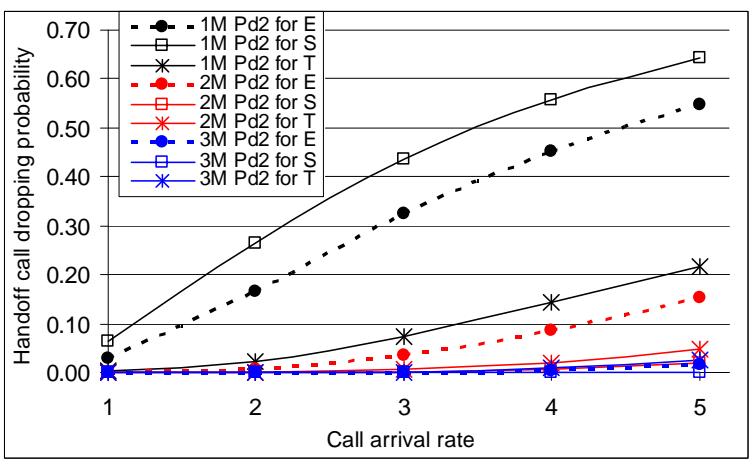

Figure 26. HCDP (Pd2) for class-2 calls with 1 M:2 M:3 M $=25: 25: 50$.

heterogeneous mobile terminals. The objectives of the proposed TJCAC algorithm are to reduce call blocking/ dropping probability, and ensure fairness in allocation of radio resources among heterogeneous mobile terminals. An analytical model has been developed for the proposed JCAC scheme in order to evaluate its performance. Performance of the proposed TJCAC scheme is compared with the performance of EJCAC and SJCAC schemes. Results show that the proposed JCAC scheme achieves lower call blocking/dropping probability for low-capability mobile terminals in the network and thereby reduces the problem of unfairness in allocation of radio resources.

\section{REFERENCES}

[1] S. Frattasi, H. Fathi, F. H. P. Fitzek, R. Prasad and M. D. Katz, "Defining 4G Technology from the User's Perspective,” IEEE Network, Vol. 20, No. 1, 2006, pp. 35-41.

[2] O. E. Falowo and H. A. Chan, "Joint Call Admission Control Algorithms: Requirements, Approaches, and Design Considerations," Computer Communications, Vol. 31, No. 6, 2008, pp. 1200-1217. doi:10.1016/j.comcom.2007.10.044

[3] D. Niyato and E. Hossain, "Noncooperative Game-Theoretic Framework for Radio Resource Management in 4G Heterogeneous Wireless Access Networks,” IEEE Transations on Mobile Computing, Vol. 7, No. 3, 2008, pp. 332-345. doi:10.1109/TMC.2007.70727

[4] X. Gelabert, J. Perez-Romero, O. Sallent and R. Agusti, "A Markovian Approach to Radio Access Technology Selection in Heterogeneous Multiaccess/Multiservice Wireless Networks," IEEE Transactions on Mobile Computing, Vol. 7, No. 10, 2008, pp. 1257-1270. doi:10.1109/TMC.2008.50

[5] A. Hasib and A. O. Fapojuwo, "Analysis of Common Radio Resource Management Scheme for End-to-End QoS Support in Multiservice Heterogeneous Wireless Networks," IEEE Transactions on Vehicular Technology, Vol. 57, No. 4, 2008, pp. 2426-2439. doi:10.1109/TVT.2007.912326

[6] Y. P. Chen and Y. H. Yang, “A New 4G Architecture 
Providing Multimode Terminals Always Best Connected Services," IEEE Wireless Communications, Vol. 14, No. 2, 2007, pp. 36-41. doi:10.1109/MWC.2007.358962

[7] W. Song and W. Zhuang, "Multi-Service Load Sharing for Resource Management in the Cellular/WLAN Integrated Network," IEEE Transactions on Wireless Communications, Vol. 8, No. 2, 2009, pp. 725-735. doi:10.1109/TWC.2009.070455

[8] S. Lee, K. Sriram, K. Kim, Y. Kim and N. Golmie, "Vertical Handoff Decision Algorithms for Providing Optimized Performance in Heterogeneous Wireless Networks," IEEE Transactions on Vehicular Technology, Vol. 58, No. 2, 2009, pp. 865-881. doi:10.1109/TVT.2008.925301

[9] S. Y. Hui and K. H. Yeung, "Challenges in the Migration to 4G Mobile Systems," IEEE Communications Magazine, Vol. 41, No. 12, 2003, pp. 54-59. doi:10.1109/MCOM.2003.1252799

[10] S. J. Lincke, "Vertical Handover Policies for Common Radio Resource Management," International Journal of Communication, Vol. 18, No. 6, 2005, pp. 527-543. doi:10.1002/dac.715

[11] W. Zhang, "Performance of Real-Time and Data Traffic in Heterogeneous Overlay Wireless Networks," Proceedings of the 19th International Teletraffic Congress, Beijing, 29 August-2 September 2005.

[12] A. Pillekeit, F. Derakhshan, E. Jugl and A. MitscheleThiel, "Force-Based Load Balancing in Co-Located UMTS/ GSM Networks,” IEEE 60th Vehicular Technology Conference, Vol. 6, 2004, pp. 4402-4406.

[13] K. H. Suleiman, H. A. Chan and M. E. Dlodlo, "Load Balancing in the Call Admission Control of Heterogeneous Wireless Networks," International Conference on Communications and Mobile Computing (IWCMC'06), Vancouver, 3-6 July 2006, pp. 245-250.

[14] J. Perez-Romero, O. Sallent, R. Agusti, L. Wang and H. Aghavmi, "Network-Controlled Cell-Breathing for Capacity Improvement in Heterogeneous CDMA/TDMA Scenarios," IEEE Wireless Communications and Networking Conference (WCNC'06), Las Vegas, 3-6 April 2006, pp. 36-41.

[15] O. E. Falowo and H. A. Chan, "Joint Call Admission Control for Next Generation Wireless Networks," Proceedings of IEEE Canadian Conference on Electrical and Computer Engineering, Ottawa, 7-10 May 2006, pp. 11511154.

[16] R. B. Ali and S. Pierre, “An Efficient Predictive Admission Control Policy for Heterogeneous Wireless Bandwidth Allocation in Next Generation Mobile Networks," International Conference on Communications and Mobile Computing (IWCMC’06), Vancouver, 3-6 July 2006.

[17] L. Chen, P. Wang, M. Song, Y. Man and J. Liu, “A Novel Load Balancing Scheme for QoS Provisioning over Heterogeneous Wireless Networks,” 6th International Conference on Pervasive Computing and Applications (ICPCA), 26-28 October 2011.

[18] J. Sun, X. L. Wu and X. J. Sha, "Load Balancing Algo- rithm with Multi-Service in Heterogeneous Wireless Networks," 6th International ICST Conference on Communications and Networking in China, Harbin, 17-19 August 2011, pp. 703-707.

[19] O. E. Falowo and O. Olowole, "Effect of RAT Selection Based on Service Symmetry and Network Duplex Mode in Heterogeneous Wireless Networks," IEEE Africon 2011-The Falls Resort and Conference Centre, Livingstone, 13-15 September 2011, pp. 1-6.

[20] G. Fettweis, "Current Frontiers in Wireless Communications: Fast \& Green \& Dirty,” IEEE Wireless Communications \& Networking Conference (WCNC), Budapest, 5-8 April 2009.

[21] H. Holma and A. Toskala, "WCDMA for UMTS," 2nd edition, John Wiley \& Sons, New York, 2001.

[22] V. Pla, J. M. Gimenez-Guzmany, J. Martinez and V. Casares-Giner, "Optimal Admission Control Using Handover Prediction in Mobile Cellular Networks," Proceedings of the 2nd International Working Conference on Performance Modelling and Evaluation of Heterogeneous Networks (HET-NETs ’04), Ilkley, 26-28 July 2004.

[23] G. Kesidis, J. Walrand and C.-S. Chang, "Effective Bandwidths for Multi-Class Markov Fluids and Other ATM Sources," IEEE/ACM Transactions on Networking, Vol. 1, No. 4, 1993, pp. 424-428. doi:10.1109/90.251894

[24] M. Gabowski, M. Stasiak, A. Wisniewski and P. Zwierzykowski, "Uplink Blocking Probability Calculation for Cellular Systems with WCDMA Radio Interface, Finite Source Population and Differently Loaded Neighbouring Cells," Proceedings of the 11th Asia-Pacific Conference on Communications (APCC'05), Perth, 3-5 October 2005, pp. 138-142.

[25] N. Nasser and H. Hassanein, "Dynamic Threshold-Based Call Admission Framework for Prioritized Multimedia Traffic in Wireless Cellular Networks," Proceedings of the IEEE Global Telecommunications Conference (GLOBECOM'04), Dallas, Texas, 29 November-3 December, 2004, pp. 644-649.

[26] S.-P. Chung and J.-C. Lee, "Performance Analysis and Overflowed Traffic Characterization in Multi-Service Hierarchical Wireless Networks," IEEE Transactions on Wireless Communications, Vol. 4, No. 3, 2005, pp. 904 918. doi:10.1109/TWC.2005.847031

[27] P. V. Orlik and S. S. Rappaport, "On the Handover Arrival Process in Cellular Communications," ACM/Baltzer Wireless Networks, Vol. 7, No. 2, 2001, pp. 147-157. doi:10.1023/A:1016685506058

[28] D. P. Bertsekas and J. N. Tsitsiklis, "Introduction to Probability,” Athena Scientific, Belmont, 2002.

[29] O. E. Falowo and H. A. Chan, "Joint Call Admission Control Algorithm for Fair Radio Resource Allocation in Heterogeneous Wireless Networks Supporting Heterogeneous Mobile Terminals," 7th Annual IEEE Consumer and Communication \& Networking Conference (IEEE CCNC), Las Vegas, 9-12 January 2010, pp. 1-5. 